\title{
Invasão de áreas de savana intra-amazônicas por Digitonthophagus gazella (Fabricius, 1787) (Insecta: Coleoptera: Scarabaeidae)
}

\author{
Rodrigo Augusto MATAVELLI ${ }^{1}$, Julio Neil Cassa LOUZADA²
}

RESUMO

Neste estudo, foi avaliada a invasão da comunidade de Scarabaeinae detritívoros de uma savana amazônica pela espécie africana Digitonthophagus gazella (Fabricius 1787). O estudo foi realizado nas proximidades da vila de Alter do Chão (2o $31^{\prime}$ S e $55^{\circ}$ $\left.00^{\prime} \mathrm{W}\right)$, localizada a aproximadamente $36 \mathrm{~km}$ a sudoeste de Santarém, Pará, Brasil. Vinte e duas áreas de savanas de $3,75 \mathrm{ha}$ (250 × $150 \mathrm{~m}$ ) distribuídas em 30.000 ha foram amostradas, no período de 21 de julho a 13 de agosto de 2003 , utilizando 66 armadilhas de queda com três tipos de iscas (fezes bovinas, fezes humanas e carcaças). Foram encontrados indivíduos de $D$. gazella em quatro das vinte e duas áreas amostradas. Procurou-se explicar a presença da espécie nas áreas de savana através de análises de regressão logística, onde as variáveis explicativas foram: ocorrência de queimada nos últimos seis anos, diversidade e abundância total de Scarabaeidae nativos presentes na área, abundância de Canthon sp.1, (espécie de Scarabaeidae mais abundante na região). Exceto pela abundância total de indivíduos de Scarabaeidae nativos, nenhuma das variáveis bióticas e abióticas tiveram efeito estatisticamente significativo na presença do D. gazella. Estes resultados podem ser explicados por: (a) algum fator ainda não analisado, relacionado à invasão da área pelo D. gazella; (b) Não houve tempo para a dispersão e estabelecimento da espécie em todas as áreas; (c) A comunidade nativa de Scarabaeinae apresenta resistência à invasão pelo D. gazella.

PALAVRAS-CHAVE: Amazônia, Ecossistema tropical, Brasil, Invasão biológica.

\section{Invasion of intra-Amazonian savannas by the dung beetle Digintonthophagus gazella (Fabricius, 1787) (Insecta: Coleoptera: Scarabaeidae)}

\section{ABSTRACT}

This work aimed to verify the invasion of the dung beetles community from intra-Amazonian savanna by the African species Digitonthophagus gazella (Fabricius 1787). The research was carried out near Alter do Chão village (2 $31^{\prime}$ S; 550 $00^{\prime}$ W), 36 Km Southwest of Santarém, Pará, Brazil. Twenty two areas were sampled, from 21 July to 13 August 2003, using 66 baited pitfall traps (cattle dung, human faeces and carcass bait). D. gazella individuals were found in four of twenty two sampled areas. The presence of the species in the savanna was analysed by a logistic regression, using the occurrence of burnings in the last six years, species richness and abundance of native Scarabaeinae, and abundance of Canthon sp1, (the most abundant species in the region), as explanatory variables. Except for the total abundance of native Scarabaeinae, none of the variables were statistically significant for the presence of D. gazella. Hypothetically, these results were probably due to a non-analysed factor related to the invasibility of the area by D. gazella, or lack of time for the species to disperse and establish in all the areas, or a resistance of native dung beetle community to $D$. gazella invasion. Nevertheless, there must be a competitive mechanism of invasion resistance bounding the abundance of native species and D. gazella invasibility.

KEYWORDS: Amazonian, Tropical ecosystem, Brazil, Biological invasion.

1 Estudante de pós-graduação da Universidade Federal de Lavras. Departamento de Biologia, Universidade Federal de Lavras, Lavras - MG. CEP $37200-000$. e-mail: ram_eco@yahoo.com.br;

2 Professor, Doutor em Entomologia pela Universidade Federal de Viçosa. Departamento de Biologia, Universidade Federal de Lavras, Lavras - MG. CEP 37200-000 e-mail: jlouzada@ufla.br (corresponding author) 


\section{INTRODUÇÃO}

A sub-família Scarabaeinae é uma das mais diversificadas em espécies da ordem Coleoptera. Os insetos desta família apresentam hábitos detritívoros e estão presentes em todos os ecossistemas terrestres brasileiros, incluindo o Cerrado (Milhomen et al., 2003).

Os Scarabaeinae são considerados organismos importantes na manutenção sanitária dos ecossistemas pelo fato de utilizarem com grande eficiência fezes de vertebrados, carcaças e matéria orgânica em decomposição como alimento (Halffter \& Mattheus, 1996). Sua interação com o ambiente os credencia como importantes inimigos naturais de moscas, parasitas e demais insetos que utilizam detritos com recurso alimentar ou vetor de dispersão (Ridsdill-Smith, 1980).

A modificação de habitats naturais através de distúrbios nos ambientes abiótico e biótico pode levar a alteraçōes na estrutura e composição das comunidades. Estas alterações podem resultar no declínio de populaçóes nativas e mesmo na extinção de espécies (Pimm, 1986).

Invasões de espécies exóticas em um novo território são excelentes objetos de estudos acadêmicos em ecologia, mas os efeitos que esses processos têm na estrutura das comunidades nativas brasileiras são pouco compreendidos. As invasōes biológicas são consideradas o segundo principal agente de perda da biodiversidade em escala mundial (Glowka et al., 1994, Lonsdale, 1999).

Em diversos países, desenvolveram-se nas ultimas décadas projetos envolvendo besouros coprófagos como agentes de ciclagem de nutrientes e melhoradores da condição física do solo, destruidores de parasitas gastrointestinais de ruminantes e competidores de moscas que reproduzem na massa fecal (Doube et al, 1991). Estes projetos levaram à introdução de espécies de Scarabaeinae, principalmente africanas, em diversas partes do mundo, como Brasil, Chile, Havaí, Estados Unidos e Austrália. A principal espécie introduzida foi o Digitonthophagus gazella (Fabricius 1787).

Digitonthophagus gazella, anteriormente considerado como pertencente ao gênero Onthophagus Latreille 1802, é uma espécie amplamente distribuída em regiōes tropicais da África, incluindo Madagascar, e na península da Arábia, Índia e Sri Lanka. Essa espécie foi introduzida no Havaí em 1958 (Harris et al, 1982), na Austrália em 1968 (Bornemissza 1976) e, em diferentes etapas no Chile (Zunino \& Barbero, 1993), nos Estados Unidos (Blume \& Aga 1978; Fincher et al, 1983) e no Brasil (Miranda et al., 1990; Bianchin et al, 1998). A América Central tem sido invadida pelo $D$. gazella por indivíduos das populações introduzidas inicialmente nos Estados Unidos (Barbero \& López-Guerrero, 1992). No Brasil a espécie tem sido estudada para fins de controle biológico de moscas e incorporação de matéria orgânica no solo. Nesses estudos foram avaliados dados bionômicos da espécie (Fonseca \& Kerr, 2005), seu efeito na melhoria da fertilidade dos solos (Galbiati et al, 1995; Miranda et al, 1998), sua sazonalidade (Koller et al, 1997; Aidar et al, 2000) e sua interação antagônica com estratégias de controle químico de moscas (Bianchin et al, 1998; Diersmann et al, 2006). Entretanto, nenhum trabalho até o presente avaliou a distribuição da espécie no Brasil e sua ocorrência em ambientes naturais.

As savanas são um dos maiores componentes da vegetação do mundo, cobrindo uma superfície de 15 milhôes de quilômetros quadrados, e contribuem com aproximadamente $30 \%$ da produção primária do planeta (IPCC, 1990). Na América do Sul, as formações savânicas ocupam 2,5 milhôes de $\mathrm{Km}^{2}$, sendo que $80 \%$ desta área são ocupadas pelo cerrado. Entretanto existem enclaves na região Amazônica de ambientes savânicos que apresentam peculiaridades tanto botânicas quanto faunísticas importantes.

Este trabalho relata pela primeira vez a invasão de áreas de savana intra-amazônica por D. gazella e explora algumas das possibilidades de explicação para a ocorrência de tal fenômeno ligado à ocorrência de queimadas e a comunidade nativa de Scarabaeinae.

\section{MATERIAL E MÉTODOS}

\section{ÁREA DE ESTUDO}

O estudo foi realizado nas proximidades da vila de Alter do Chão (2³1' S e 5500' W), situada na margem direita do Rio Tapajós próximo à confluência com o rio Amazonas. A vila está localizada a aproximadamente $36 \mathrm{~km}$ ao sudoeste do município de Santarém, no estado do Pará, Brasil.

O clima apresenta duas estaçôes: o período das chuvas, que se distribui entre os meses de janeiro e julho e o período de estiagem, que vai de julho a dezembro. A precipitação anual média na região é de $1420 \mathrm{~mm}$, e a temperatura média é de $27,7^{\circ} \mathrm{C}$.

As áreas de estudo apresentam um mosaico de vegetação peculiar com manchas de savana entre os fragmentos de floresta de terra firme e matas de igapó. As áreas de savana são cobertas principalmente por duas espécies de gramíneas: Paspalum carinatum Humb. \& Bonpl. ex Flügge e Trachypogon plumosos Humb. \& Bonpl. ex Willd., com árvores esparsas, apresentando um solo geralmente pobre e arenoso (Magnusson et al, 2001). Estudos florísticos indicaram uma semelhança entre a vegetação da savana e o cerrado do Brasil central, porém com uma menor diversidade de espécies que este último (Miranda et al., 1993).

A unidade amostral básica deste trabalho foi uma parcela que cobriu uma área de 3,75 ha $(250 \times 150 \mathrm{~m})$, contendo quatro transectos de $250 \mathrm{~m}$ espaçados $50 \mathrm{~m}$ entre si, os transectos foram estabelecidos no sentido norte-sul. Vinte 
e duas parcelas foram distribuídas de maneira sistemática dentro de uma área de 30.000 ha de forma a cobrir o mais uniformemente possível as manchas de savana.

\section{COLETA}

Para obtenção das amostras da comunidade de Scarabaeinae foi realizada uma campanha de campo no período de 21 de julho a 13 de agosto de 2003, período considerado de "estiagem" na região e que apresenta a maior atividade de insetos.

Em cada parcela foram coletados Scarabaeinae em 66 pontos amostrais posicionados a cada $50 \mathrm{~m}$ em quatro transectos paralelos de $250 \mathrm{~m}$. Os transectos foram separados uns dos outros por $50 \mathrm{~m}$ e foram posicionados a no mínimo $50 \mathrm{~m}$ da borda da floresta. A distância mínima de $50 \mathrm{~m}$ da floresta foi assumida arbitrariamente como o mínimo necessário para não se observar influência desse sistema sobre as armadilhas.

Em cada ponto foram instaladas três armadilhas, cada uma contendo uma isca atrativa (fezes bovinas, fezes humanas ou fígado bovino apodrecido), que são amplamente utilizadas em estudos com a comunidade de Scarabaeinae. As armadilhas foram constituídas de potes plásticos com $10 \mathrm{~cm}$ de altura e 15 $\mathrm{cm}$ de diâmetro, enterrados com a abertura no nível do solo. As iscas foram fixadas no centro da abertura de cada pote. Dentro das armadilhas foram colocados aproximadamente $40 \mathrm{ml}$ de solução de formol $1 \%$ e seis gotas de detergente para quebrar a tensão superficial da água. As armadilhas foram colocadas a campo pela manha e coletadas 24 horas depois.

O material coletado foi triado e conservado em álcool $70 \%$. Os insetos foram levados para o laboratório de Ecologia de Comunidades do Instituto Nacional de Pesquisa da Amazônia, onde foram separados em morfotipos e acondicionados para a posterior identificação taxonômica. A identificação taxonômica foi realizada por comparação com a coleção do Setor de Ecologia da UFLA - Universidade Federal de Lavras e auxílio de F. Z. Vaz-de-Mello.

\section{VERIFICAÇÃO DA INVASÃO POR $D$. gazella}

Para testar a possível influência de fatores bióticos e abióticos na presença de $D$. gazella nas áreas de savana intraamazônica, foram executadas análises de regressão logística, onde a variável resposta foi a presença ou ausência da espécie invasora e as variáveis determinantes foram: (a) Número total de espécies da comunidade de Scarabaeinae nativos; (b) Número total de indivíduos da espécie mais abundante da comunidade nativa (Canthon sp.1); (c) Número de vezes que a área foi submetida à queimada no período de 1997 a 2002 (sem dados disponíveis para o ano de 1998).

\section{RESULTADOS E DISCUSSÃO}

Indivíduos de $D$. gazella foram encontrados em quatro das vinte duas áreas de savanas intra-amazônicas estudadas. A influência de variáveis bióticas e abióticas sobre a presença ou ausência do D. gazella foi não significativa para a maioria das variáveis explicativas propostas.

Não foi observado nenhum efeito significativo da freqüência de queimadas na área de savana sobre a invasão do local pelo D. gazella $\left(\chi^{2}=0,012, \mathrm{p}=0,913\right)$. Nenhuma relação foi encontrada entre a invasão do $D$. gazella e o tamanho populacional da espécie mais abundante, Canthon sp1 $\left(\chi^{2}=0,583 \mathrm{p}=0,44484\right)$. O número total de espécies de Scarabaeinae nativos presentes na área não influenciou a presença ou ausência de D. gazella.

Entretanto, o número total de indivíduos de espécies nativas da comunidade presentes nas áreas de savana afetou negativamente a invasibilidade ou presença do D. gazella $\left(\chi^{2}\right.$ $=4,597, \mathrm{p}=0,032)$.

Das vinte e duas áreas de savana intra-amazônicas analisadas somente quatro apresentaram $D$. gazella como parte da comunidade de Scarabaeinae detritívoros. Aparentemente a espécie encontra-se ainda na fase de estabelecimento nestas áreas. A abundância ainda é baixa, se comparada a várias espécies nativas pertencentes à mesma comunidade (Tabela $1)$.

O fato de que a presença de $D$. gazella na área de savana não se correlacionou com a maioria dos fatores bióticos e abióticos analisados, pode estar associado a quatro possibilidades alternativas de explicação: (a) Algum fator ainda não analisado, tal como a presença de gado nas proximidades ou mesmo no interior da área, ou densidade da vegetação, pode estar relacionado à invasibilidade da área por D. gazella; (b) Não houve tempo para que a espécie se disperse e se estabeleça em todas as áreas; (c) a comunidade nativa apresenta resistência à invasão por $D$. gazella.

A presença de $D$. gazella nas áreas de savana foi influenciada negativamente pela abundância média das espécies nativas da área, o que pode indicar uma resistência natural da comunidade de Scarabaeinae nativos a invasão por $D$. gazella. A ausência de relação entre a invasão por espécies exóticas e a diversidade de espécies tem sido mostrada em outros sistemas (Melinda et al., 2004), onde também as relaçôes de abundância se mostraram mais ligadas à possibilidade ou não de invasão.

As comunidades de Scarabaeinae respondem prontamente a modificaçōes ambientais através de alteraçōes de riquezas de espécies, distribuição de abundâncias, composição específica ou na estrutura das guildas (Halffter \& Favila, 1993). Nossos dados sugerem que as comunidades nativas de Scarabaeinae das savanas intra-amazônicas são susceptíveis a invasão por $D$. 


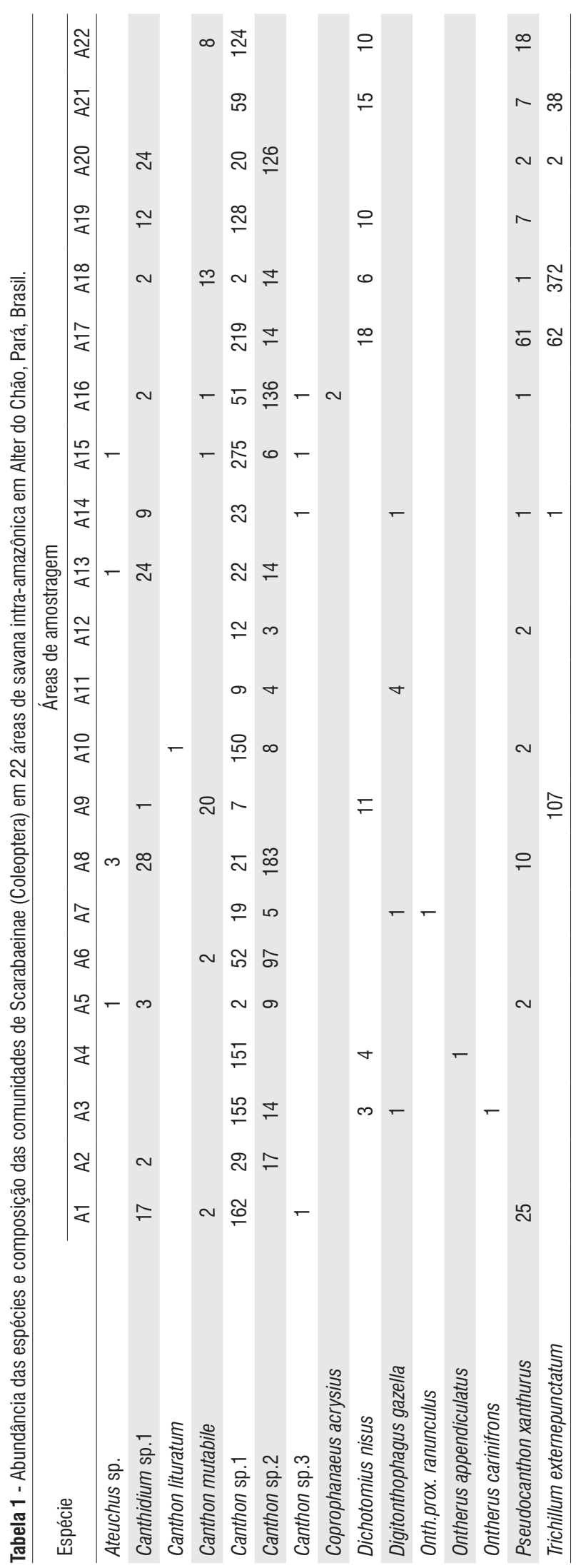

gazella caso algum fator altere a abundância média das espécies. Este fato pode estar relacionado à diminuição da pressão competitiva exercida por parte das espécies residentes.

Digitonthophagus gazella é uma espécie altamente adaptada a fezes bovinas e de grandes herbívoros (Rougon \& Rougon, 1980). Este fato pode ajudar na explicação dos padrões encontrados, já que aparentemente as espécies nativas são mais eficientes na utilização dos recursos disponíveis nas savanas intra-amazônicas. Até o momento da aquisição dos dados deste trabalho as áreas de savana analisadas não apresentavam o gado bovino como elemento faunístico, o que pode ajudar a explicar a baixa abundância da espécie nas áreas onde foi encontrada.

Digitonthophagus gazella é uma espécie típica de ambientes abertos e com uma rápida taxa de reprodução (Rougon \& Rougon, 1980; Lee \& Peng 1981, 1982) apresentando estratégia oportunista de ocupação (Cambefort, 1984) e com uma rápida mobilidade individual (Seymour, 1980). Esta espécie apresenta taxas de dispersão que oscilam entre $30 \mathrm{e}$ $90 \mathrm{Km}$ /ano (Rivera-Cervantes \& Garcia-Real, 1991; Barbero \& López-Guerrero, 1992). Entretanto, sua dispersão pode ser influenciada por fatores inerentes das paisagens (florestas, corpos d'água etc.) e características das comunidades de Scarabaeinae já instaladas no local. Onde é nativa, a população pode ser inibida devido à competição por recursos com outros besouros coprófagos, pelo cleptoparasitismo ou predação (Rougon, 1987).

Aparentemente, a invasão de $D$. gazella em áreas de savanas intra-amazônicas ainda está em sua fase inicial e a comunidade nativa ainda apresenta resistência à invasão. Entretanto a progressiva ação antrópica nestes sistemas pode levar à diminuição das populaçōes nativas ou alterar a quantidade e qualidade dos recursos disponíveis, o que pode facilitar a invasibilidade pela espécie. Estudos futuros de monitoramento a longo prazo das comunidades de Scarabaeinae de savanas intra-amazonicas poderão confirmar o status do processo de invasão pela espécie exótica e seu risco sobre a biodiversidade deste sistema.

\section{AGRADECIMENTOS}

Agradecemos à Dra Albertina Pimentel Lima pelo suporte técnico científico na aquisição dos dados deste trabalho e ao Dr. Fernando Z. Vaz-de-Mello e ao Sr. Ayr de Moura Bello pela identificação das espécies de Coleoptera e a família de Deco e Juci, principalmente ao Welliton, pela colaboração nas coletas de campo. Agradecemos também a três revisores anônimos pelas suas valiosas críticas e sugestōes. 


\section{BIBLIOGRAFIA CITADA}

Aidar, T.; Koller, W.W.; Rodrigues, S.R.; Corrêa, A.M.; Silva, J.C.C.; Balta, O.S.; Oliveira, J.M. \& Oliveira, V.L. 2000. Besouros coprófagos (Coleoptera; Scarabaeidae) coletados em Aquidauana, MS, Brasil. Anais Sociedade Entomologica. Brasileira, 29(4): 817-820.

Barbero, E.; López-Guerrero, Y. 1992. Some considerations on the dispersal power of Digitonthophagus gazella (Fabricius, 1787) in the new world (Coleoptera, Scarabaeidae, Scarabaeinae). Tropical Zoology, 5(1): 115-120.

Bianchin, I.; Alves, R.G.O.; Koller, W. 1998. Efeito de carrapaciticidas/ inseticidas "pour on" sobre adultos de besouro coprófago africano Onthophagus gazella Fabr. (Coleoptera. Scarabaeidae). Anais da Sociedade Entomologica do Brasil, 27(3): 275-279.

Bornemissza, G. E. 1976. The Australian dung beetle project 1965-1975. Australian Meat Research Committee Review, 30(1): $1-30$.

Blume, R. R.; Aga. A. 1978. Onthophagus gazella F: progress of experimental release in south Texas. Folia entomológica Mexicana, 29-40(1): 190-191.

Cambefort, Y. 1984. Étude écologique des coléoptères Scarabaeidae de Côte d'Ivoire. Travaux des Chercheurs de la Station de Lamto (Côte d'Ivoire), 3(1): 1-320.

di Castri, F.A.; Hansen J.; Debussche, M. 1990. Biological invasions in Europe and the Mediterranean Basin. Monographiae Biologicae n 65. 76pp.

Diersmann, E.M.; Ferreira, L.R.; Chaaban, A.; Moya-Borja, G.E. 2006. Impacto do uso de ivermectinas de longa ação na sucessão ecológica dos excrementos de bovinos tratados, tomando-se como modelo o besouro coprófago Digitonthophagus gazella (Fabricius, 1787). Revista Brasileira de Agroecologia, 1(1): 883-886.

Doube, B.M.; Macqueen, A.; Ridsdill-Smith T.J.; Weir, T.A. 1991. Native and introduced dung beetles in Australia. In: Hanski, I.; Cambefort, Y. (eds.). Dung beetle ecology. Princeton University Press, Princeton. p. 255-278.

Fincher, G. T.; Stewart, T. B.; Hunter III, J. S. 1983. The 1981 distribution of Onthophagus gazella Fabricius from releases in Texas and Onthophagus taurus Schreber from an unknown release in Florida (Coleoptera. Scarabaeidae). Coleopterists Bulletin, 37(2): 159-163.

Fonseca, M.O.; Kerr, W.S. 2005. Avaliação de índices reprodutivos em besouro africano (Digitontophagus gazella Fabricius) (Coleoptera: Scarabaeidae). Bioscience Journal, 21(3): 61.68.

Galbiati, C.; Bensi, C.; Conceição, C.H.C.; Florcoviski, J.F. \& Calafiori, M.H. 1995. Estudo comparativo entre besouros do esterco Dichotomius anaglypticus (Mann., 1829) e Onthophagus gazella (F.), sobre a pastagem, em condições brasileiras. Ecossistema, 20(3): 109-118.

Glowka, L.; Burhenne-Guilmin, F.; Synge, H. 1994. A Guide to the Convention on Biological Diversity, Gland: IUCN. 256pp.

Halffter, G. Matthews. E. 1996. The natural history of dung beetles of the family Scarabaeinae (Coleoptera: Scarabaeidae). Folia Entomológica Mexicana. 12-14(1): 1-312.
Halffter, G.; Favila, M.E. 1993. The Scarabaeinae (Insecta: Coleoptera): an animal group for analyzing, inventorying and monitoring biodiversity in tropical rainforest and modified landscapes. Biology International, 27(1): 15-21.

Harris, R.L.; Onaga, K.; Blume, R.R.; Roth, J.P.; Summerlin, J.W. 1982. Survey of beneficial insects in undisturbed cattle droppings on Oahu, Hawaii. Proceedings of the Hawaiian Entomological Society, 24 (1): 91-95.

IPCC, 1990. Climate change: the IPCC Scientific Assessment. Cambridge Press, London. 231pp.

Koller, W.W.; Gomes, A.; Flechtmann, C.A.H.; Rodrigues, S.R.; Bianchin, I.; Honer, M.R. 1997. Ocorrência e sazonalidade de besouros copro/necrófagos (Coleoptera; Scarabaeidae), em massas fecais de bovinos, na região de Cerrados do Mato Grosso do Sul.: EMBRAPA-CNPGC, Campo Grande. Pesquisa em Andamento, 48, 5 pp.

Lee, J.M.; Peng, Y.S. 1981. Influence of adult size Onthophagus gazella on manure pad degradation, nest construction, and progeny size. Environmental Entomology, 10(5): 626-630.

Lee, J.M.; Peng, Y.S. 1982. Influence of manure availability and nesting density on the progeny size of Onthophagus gazella. Environmental Entomology, 11(1): 38-41.

Lodge, D.M.; 1993. Biological invasions: Lessons for ecology. Trends in Ecology and Evolution, 8(2): 133-137.

Lonsdale, W.M. 1999. Global patterns of plant invasions and the concept of invasibility. Ecology, 80(5): 1522-1536.

Magnusson, W.E.; Lima, A.P.; Faria, A.S.; Victoria, R.L.; Martinelli, L.A. 2001. Size and carbon acquisition in lizards from Amazonian savanna: evidence from isotope analysis. Ecology, 82(6): 1772-1780.

Melinda, D.S.; Wilcox, J.C.; Kelly, T.; Knapp, A.K. 2004. Dominance not richness determines invasibility of tallgrass prairie. Oikos, 106(2): 253-262.

Milhomem, M.; Vaz-de-Melo, F.Z.; Diniz, I.R. 2003. Técnicas de coleta de besouros copronecrófagos no Cerrado. Pesquisa Agropecuária Brasileira, 38(11): 1249-1256.

Miranda, C.H.B.; do Nascimento, Y.A.; Bianchin, A. 1990. Desenvolvimento de um programa entregado de controle dos nematódeos e a mosca-dos-chifres na região dos cerrados. Fase 3. Potencial de Onthophagus gazella no enterrio de fezes bovinas. EMBRAPA-gado de corte, Pesquisa em Andamento, 42: 1-5.

Miranda, C.H.B.; Santos, J.C.C.; Bianchin, I. 1998. Contribuição de Onthophagus gazella à melhoria da fertilidade do solo pelo enterrio de massa fecal bovina fresca. Revista Brasileira Zootecnia, 27(5): 681-685.

Miranda, A.C.; Miranda, H.S.; Dias, I.F.O.; Dias, B.F.S. 1993. Soil and air temperatures during prescribed cerrado fires in central Brazil. Journal of Tropical Ecology, 9(3): 313-320.

Mooney, H.A.; Drake, J.A. 1986. Ecology of Biological invasions of north America and Hawaii. Ecological Studies 58. 233pp.

Ridsdill-Smith, T.J. 1980. Stages da bush fly Musca vetustíssima Walker (Diptera: Muscidae) killed by Scarabaeinae dung beetles (Coleoptera: Scarabaeidae) in favourable catlle dung. Bulletin of Entomological Research, 71(5): 425-433. 
Rivera-Cervantes, L.E.; Garcia-Real, E. 1991. New locality records for Onthophagus gazella Fabricius (coleoptera. Scarabaeidae) in Jalisco, México. Colepterists Bulletin, 37(2): 159-163.

Rougon, C.; Rougon, D. 1980. Contribuition à la Biologie des Coléopteres coprophages en region sahélienne. Ètude du développement d'Onthophagus gazella (Coleoptera: Scarabaeidae). Reevue d' Ecologie et de Biologie du sol, 17(3): 379-39.

Rougon, D. 1987. Coléopteres coprophiles en zone sahélienne: étude biocénotique, comportement nidificateur, intérvention dans le recyclage de la matiére organique du sol. Thése d'Ètat, Université d'Orléans, 324pp.

Pimm, S.L. 1986. Community structure and stability. In: Soulé, M. (ed.). Conservation biology: The science of scarcity and diversity. Sinauer, Sunderland, Massachusetts, MA, USA. p. 309-329.
Seymour, J. 1980. Dung Beetles get a little help from their friends. Ecos, 26(1): 20-25.

Smith, M.D.; Wilcox, J.C.; Kelly, T.; Knapp, A.K. 2004. Dominance not richness determinates invisibility of tallgrass praire. Oikos, 106(2): 253-262.

Zunino, M.; Barbero, E. 1993. Escarabajos, ganado, pastizales: algunas consideraciones deontológicas. Folia Entomológica Mexicana, 87(1): 95-101.

Recebido em 23/01/2007

Aceito em 01/10/2007 